$\xi=-1$

\title{
Dual Solutions in the Boundary Layer Flow and Heat Transfer in the Presence of Thermal Radiation with Suction Effect
}

\author{
Siti Nur Aisyah Azeman* \\ Faculty Computer and Mathematical Sciences, Universiti Teknologi MARA, Perak Branch, Tapah Campus, Tapah Road, \\ 35400 Perak, Malaysia \\ *Corresponding author E-mail: sitin084@perak.uitm.edu.my
}

\begin{abstract}
The dual solutions in the boundary layer flow and heat transfer in the presence of thermal radiation is quantitatively studied. The governing partial differential equations are derived into a system of ordinary differential equations using a similarity transformation, and afterward numerical solution obtained by a shooting technique. Dual solutions execute within a certain range of opposing and assisting flow which related to these numerical solutions. The similarity equations have two branches, upper or lower branch solutions, within a certain range of the mixed convection parameters. Further numerical results exist in our observations which enable to discuss the features of the respective solutions.
\end{abstract}

Keywords: Dual Solution; Similarity Transformation; Boundary Later Flow; Heat Transfer; Thermal Radiation

\section{Introduction}

Free, forced and mix convection flow are found in a few practical application that hug receivers center solar revealed in imitation of modern-day regarding wind, digital gadget cooled by fans, nuclear reactor calm all over emergency termination and friendliness converter stationed during low velocity environment. This process occur when the effects of buoyancy forces in forced convection or the effects of forced flow in free convection become significant was reported by [1]. The interaction on forced or free convection is mainly suggested within situations where the forced flow velocity is low and/or the temperature variations are large. During decade showing much convection flow analysis that is mixed at one viscous and incompressible fluid at one surface vertical was presented. Numerical solution and analysis for temperature and velocity field for consistent surface heat fluxes has been obtained by [2]. The flow driven whether by one prescribed surface temperature or by consistent surface heat flux or by Newtonian heating from corrugated surface are considered by modeling the convective flow learned previously by [3] or by convective surface boundary condition [4].

In a number of important problems in fluid mechanic and heat transfer there are equations of fundamental physical law known as partial differential equations and the purpose of theoretical work is to find the exact solution of this equation. Solutions on certain equations of partial differential equations happen in areas applied can be discovered fairly without problems in spite of the failure in that larger often methods classical to produce decisions. According to [5] found that the solutions are generally determined as similarity solutions proved by the important of certain options for those problems by using transformations of the variables, which provide limit to existing system that is on partial differential equations in accordance to one system related with ordinary differential equations. The mixed convection boundary layer flow near point lower stagnation at a circular cylinder horizontal with a sec- ond order slip velocity model and consistent surface heat flux has been considered in a recently paper by [6]. Transformation of ordinary differential (similarity) equations had been resolved in terms of the number to governing parameter other for different values. Numerical research equipped with derivation of an analysis of time-dependent to execute solution branches stability. In expanded the paper by [7] for permeable plate case, i.e. when there is suction or injection (blowing) through the wall. In addition, the dual solution with convection has gained much attention by researchers due to wide range of solutions in computations such as [8-9]. Since then, the interest of this research has been coined to study dual solution as approach for heat transfer in opposing and assisting flows by [10]. In [11] is a well-known person that discovered there are dual solutions exist in both flows which are assisting and opposing flows while doing his research in mixedconvection flow over a horizontal surface and others. Meanwhile, in [12] examined numerically dual solution in laminar mixed convection for the both flows (assisting and opposing) past a permeable shrinking surface. They found that the opposing flow reduced the amount of skin friction and concluded that upper branch solution is stable. Four different thermal boundary conditions and heat flow patterns in mixed convection analyzed by [13] using heat lines concept for wide ranges of parameters. Numerical simulation of unsteady mixed convection in a driven cavity using an externally excited sliding lid has been studied by [14]. According to [15], correlations for heat and mass transfer in the cooperating case taking into account the effect of natural convection in porous media. The dual solutions for the upper and lower branch of the porous medium significantly affect the rate of heat transfer and mass transfer in the cavity.

The objective of this paper is to analyze the dual solutions in the boundary layer flow and heat transfer in the presence of thermal radiation is considered. The suction of a fluid through the bounding surface, as, for example, in mass transfer cooling, can substantially change the flow field and, as a consequence, have an effect on the heat transfer rate from the plate. In general, suction tends to 
increase the skin friction then heat transfer coefficients, whereas injection acts into the opposite manner obtained by [16]. Injection or withdrawal over liquid via a porous bounding heated or cooled wall is about normal interest within practical problems involving film cooling, control of boundary layers etc. This action is perform to enhance heating (or cooling) of the system then can assist to delay of transition process from laminar flow by [17]. The governing partial differential equations are reduced into a set of ordinary differential equations, then the ODEs are numerically solved with bvp4c solver.

\section{Mathematical Formulation}

Consider a steady stagnation point flow of an ambient temperature $T_{\infty}$ past a stretching surface which coincides with the plane $\mathrm{y}=0$, and the flow being in the region $\mathrm{y}>0$. It is assumed that the variable surface heat flux to be $q_{w}(x)=b x$ and that there is a free stream velocity $U(x)=a x$ flowing over the plate. Buoyant force can move like the identical path as like the flow (aiding flow), then can employment of the contrary route in imitation of the opposing flow. Under these assumptions, together with regular boundary layer and Bernoulli's approximation $U \frac{d U}{d x}=-\frac{1}{\rho} \frac{d p}{d x}$, the governing equations are

$\frac{\partial u}{\partial x}+\frac{\partial v}{\partial y}=0$

$u \frac{\partial u}{\partial x}+v \frac{\partial u}{\partial y}=v \frac{\partial^{2} u}{\partial y^{2}}+U \frac{d U}{d x}+g \beta\left(T-T_{\infty}\right)$

$u \frac{\partial T}{\partial x}+v \frac{\partial T}{\partial y}=\frac{\alpha}{\rho c_{p}} \frac{\partial^{2} T}{\partial y^{2}}-\frac{1}{\rho c_{p}} \frac{\partial q_{r}}{\partial y}$

subject to the boundary conditions

$$
\begin{aligned}
& u=0, \quad v=V_{w}, \quad-k \frac{\partial T}{\partial y}=q_{w}(x) \quad \text { at } \quad y=0, \\
& u \rightarrow U(x), \quad T \rightarrow T_{\infty} \quad \text { as } \quad y \rightarrow \infty,
\end{aligned}
$$

where $g$ is the gravitational acceleration, $\beta$ the thermal expansion coefficient, $c_{p}$ is the specific heat of the fluid at constant pressure, $v$ and $\alpha$ are the kinematic viscosity and thermal diffusivity of the fluid respectively. In order to find a similarity solution in (1) - (3), subject to boundary conditions in (4) by writing

$$
\begin{aligned}
& \eta=(U / v x)^{1 / 2} y, \quad \psi=(U v x)^{1 / 2} f(\eta) \\
& T=T_{\infty}+\frac{q_{w}}{k}\left(\frac{v x}{U}\right)^{1 / 2} \theta(\eta)
\end{aligned}
$$

where $\psi$ is the stream function defined as $u=\partial \psi / \partial y$ and $v=-\partial \psi / \partial x$, so as to identically satisfy in (1) and $k$ is the thermal conductivity. From transformation in (5) obtain

$$
u=a x f^{\prime}(\eta) \text { and } \quad v=-(v a)^{1 / 2} f(\eta)
$$

where primes denote differentiation with respect to $\eta$. Substituting in (6) into (2) and (3), the following ordinary differential equations become:

$\operatorname{Pr} f^{\prime \prime \prime}+f f^{\prime \prime}+1-f^{\prime 2}+K\left(1-f^{\prime}\right)+\lambda K \theta(+)=0$
$\frac{1}{\operatorname{Pr}}(1+N) \theta^{\prime \prime}+f \theta^{\prime}-f^{\prime} \theta=0$

where primes denote differentiation with respect to $\eta$, $\lambda=G r_{x} / \operatorname{Re}_{x}^{5 / 2}$ is the buoyancy or mixed convection parameter and $\operatorname{Pr}=v / \alpha$ is the Prandtl number. Further, $G r_{x}=g \beta q_{w} x^{4} / k v^{2}$ and $\operatorname{Re}_{x}=U x / v$ are represent the local Grashof number and the local Reynolds number. Noticed that $\lambda$ is a constant with $\lambda>0$ and $\lambda<0$ for assisting flow and opposing flows respectively, while $\lambda=0$ considered as a case of the buoyancy force is inadequate where the convection flow purely forced. The boundary conditions in (4) now become

$f(0)=f_{0}, f^{\prime}(0)=0, \theta(0)=1$

$f^{\prime}(\infty) \rightarrow 1, \theta(\infty) \rightarrow 0$

where $f_{0}=f(0)=-V_{w} /(v a)^{1 / 2} f_{0}$ is a constant with $f_{0}>0$ correspond to mass suction. In (7)-(9) reduce to those found by [18] with the condition of an unpredictable wall surface temperature for $n=1$ in the research.

The dimension of physical quantities in this work can be expressed as the skin friction coefficient $C_{f}$ and the local Nusselt number $N u_{x}$, which are defined by

$C_{f}=\frac{\tau_{w}}{\rho U^{2} / 2}, N u_{x}=\frac{x q_{w}}{k\left(T_{w}-T_{\infty}\right)}$

where the skin friction $\tau_{w}$ and the heat transfer from the plate $q_{w}$ are given by

$\tau_{w}=\mu\left(\frac{\partial u}{\partial y}\right)_{y=0}, q_{w}=-k\left(\frac{\partial T}{\partial y}\right)_{y=0}$,

with $\mu$ and $k$ being the dynamic viscosity and thermal conductivity respectively. Using the non-dimensional variables in (5), one get

$\frac{1}{2} C_{f} \operatorname{Re}_{x}^{1 / 2}=f^{\prime \prime}(0), N u_{x} / \operatorname{Re}_{x}^{1 / 2}=\theta^{\prime}(0)$

where $N u_{x}=U x / v$ is the local Reynolds number. For this paper, being limiting value to unit Prandtl number, denote as $\operatorname{Pr}=1$. Interest main physical quantity is the values of $f^{\prime \prime}(0)$ become skin friction measurement, and the non-dimensional wall temperature $\theta(0)$. The main purpose of this paper is to find how values of $f^{\prime \prime}(0)$ and $\theta(0)$ and differs in parameter of $f^{\prime \prime}(0)$ and $\theta(0)$.

\section{Results and Discussion}

The ordinary differential equations in (7) and (8), alongside boundary conditions in (9) were solved numerically by shooting technique method in Maple software together with bvp4c function implemented in MATLAB. For the shooting method technique, the results obtained by setting different initial guesses for the values of $f^{\prime \prime}(0)$ and $\theta(0)$ where all profiles satisfy the boundary conditions but with different shapes is studied. Skin friction coefficient $f^{\prime \prime}(0)$ variation and local Nusselt number $\theta(0)$ tabled in Fig. 1 and 2 respectively, both for $\operatorname{Pr}=1$. Different from decision by [19], which states existence of two solutions only for opposing flow case $(\lambda<0)$, this buoyancy of figures proof of existence of 
two solutions for both buoyancy assisting $(\lambda>0)$ and opposing $(\lambda<0)$ flows. Existences of both solutions determine by the critical value of $\lambda$, denote as $\lambda=\lambda_{c}$, which found by [19] states that there is no solution which exists for both flows.

Validity regarding two solutions illustrated in Fig. 3 and 4 are sustained by velocity and temperature profile presented in Fig. 1 and 2 respectively. Considered into this results depend on expectation there was two different profiles for parameter $\lambda$, stability equivalence where both satisfactory asymptotically far field boundary condition. Fig. 3 and 4 show the variations of the skin friction coefficient $f^{\prime \prime}(0)$ and the wall temperature $\theta^{\prime}(0)$ with buoyancy parameter $\lambda$ for value of $f_{0}=0$ (impermeable wall) and 0.5 (suction) for $\operatorname{Pr}=1$. The lower and upper branch solution are identified in the next information given on how the solution execute in Fig. 3 which shows that there is a greater value of $f^{\prime \prime}(0)$ as a solution of upper branch for a given $\lambda$ compare to lower branch solution.

According to the result, there exists a solution in assisting flow for a dual solution when the values of positive $\lambda$ are considered that much greater values of $\lambda$ proven by Fig. 3 and 4 . Results shows that that there is critical value $\left|\lambda_{c}\right|$ increases as suction $f_{0}>0$ value raised and it proves the suction $f_{0}$ increase the solution of existence in (7)-(9). The solution of lower branch become unbounded as indicated in Fig. 4 for the wall temperature $\theta(0)$ as values of $\lambda$ approaches to negatives values and also values lambda $\lambda$ to positive values. Figure 1 shows that at the upper branch solution execute a solution for the velocity profile where the velocity have positive gradient at the wall along with the opposite flow that turn out to be a region for lower branch solution. Solution of the velocity at the wall can be obtained by finding its positive for the value of $\lambda=0.8$, in Figure 6 on both branches, in agreement with the curves of $f^{\prime \prime}(0)$ proven in Figure 4.

Meanwhile, for the lower branch with value of $\lambda=0.8$, in Fig. 5 the solution has a region of reversed flow (has $f^{\prime}(\eta)<0$ for finite range of $\eta$ ) located away from the wall $(\eta=0)$. The velocity gradient at the wall is effective to find the solution regarding both branches, in agreement with the curves of $f^{\prime \prime}(0)$ as proven in Fig. 3 for $\lambda=0.8$. However, the solution on the lower branch for $\lambda=0.8$ has an area of reversed flow where $f^{\prime}(\eta)<0$ for finite range of $\eta$ situated away from the wall $(\eta=0)$. Fig. 2 $(\lambda=-0.4)$ proves that solutions for lower branch are the amount of the wall heat profiles denote as $\theta(\eta)$ are greater in contrast according to the results obtained for upper branch solutions that constant together with values in Fig. 4. The heat profiles for the solutions regarding both branches have $\theta(\eta)>0$ for all values of $0 \leq \eta<\theta$, shown in Fig. 4 whereas in Fig. 2 shows that the solution of lower branch have province below the ambient temperature, i.e. $\theta(\eta)<0$ for variation of $\eta$ and $\theta \rightarrow 0$ from below as $\eta \rightarrow \infty$.

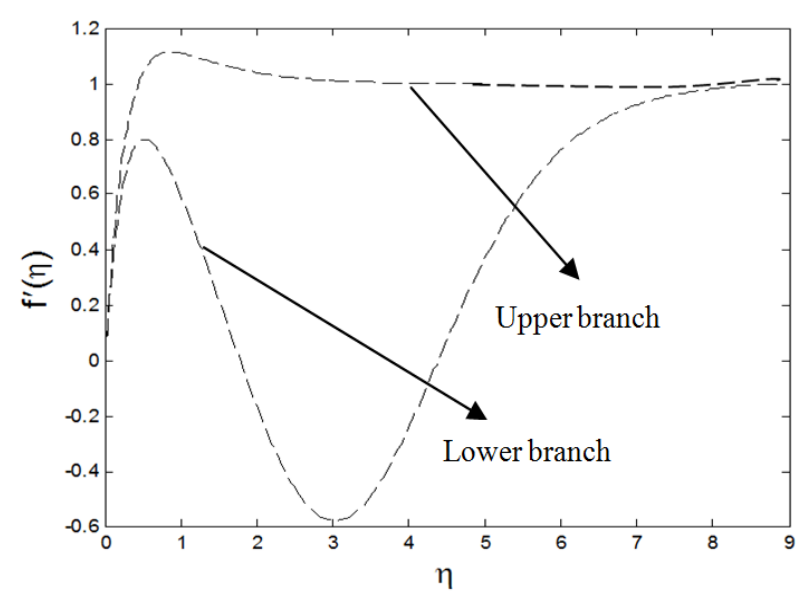

Fig. 1: Velocity profile when $\operatorname{Pr}=1$ when $\lambda=-0.4$

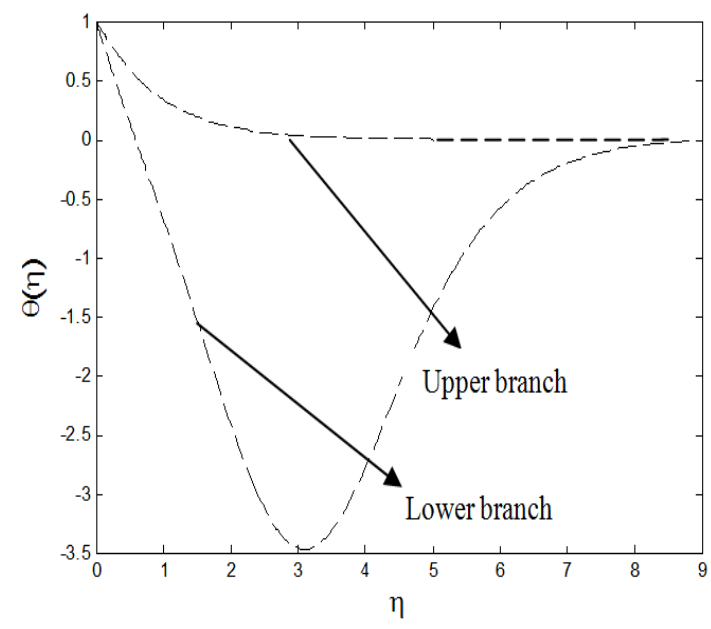

Fig. 2: Temperature profile when $\operatorname{Pr}=1$ when $\lambda=-0.4$

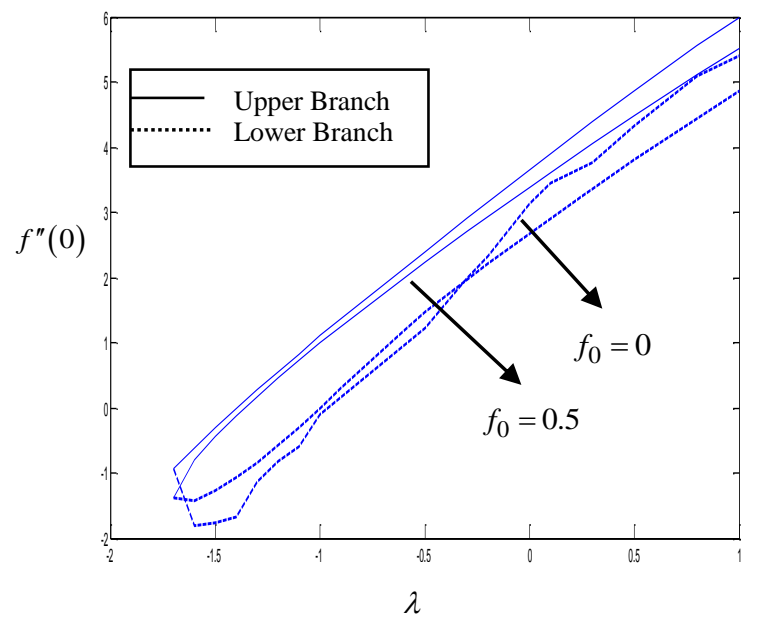

Fig. 3: The values of the skin friction coefficient $f^{\prime \prime}(0)$ with variation of $\lambda$ for $f_{0}=0.5$ and 0 for $\operatorname{Pr}=1$ 


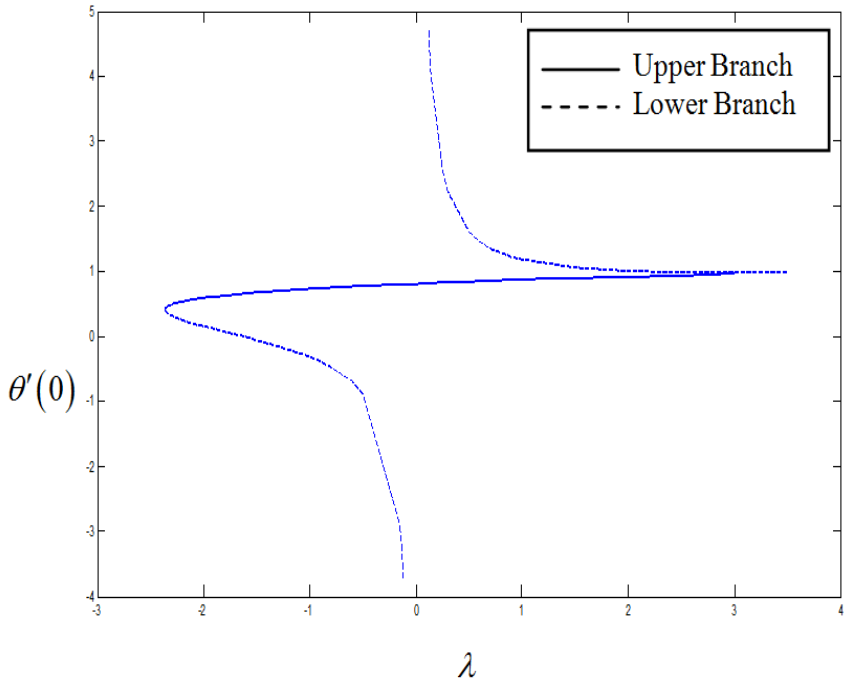

Fig. 4: The values of the wall temperature $\theta^{\prime}(0)$ with variations of $\lambda$ for $f_{0}=0.1$ with $\operatorname{Pr}=1$

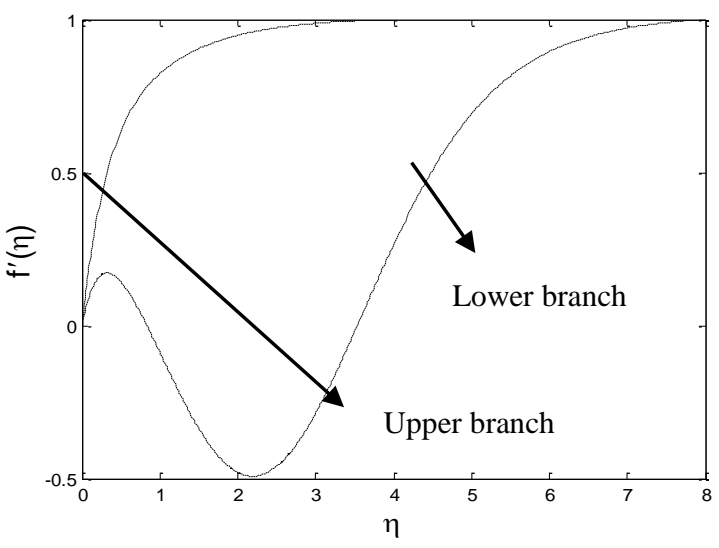

Fig. 5: Velocity profile when $\operatorname{Pr}=1$ when $\lambda=0.8$

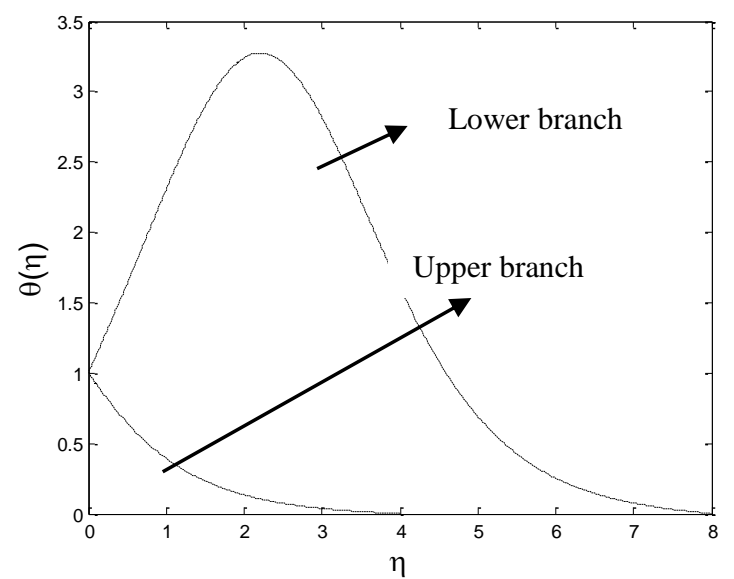

Fig. 6: Temperature profile when $\operatorname{Pr}=1$ when $\lambda=0.8$

\section{Conclusion}

Numerical preferences on momentum and energy equations are observed and it is determined that the involving parameters distinctly affected the flow area and temperature distribution. In this research, the effect of involving parameter which are mass suction parameter $f_{0}=0$ and 0.5 , Prandtl number $\operatorname{Pr}$ and the buoyancy parameter $\lambda$ on the flow field of fluid and the characteristic of heat convection $\mathrm{c}$ is discussed.

As the increment values concerning $\lambda$ increases the range of velocity values the suction delays the boundary layer separation and as a result increases the range of similarity solution. There exist dual solutions in assisting flow regime while in the opposing flow the solution terminated with critical value of $\lambda=\lambda_{c}$.

\section{Acknowledgement}

The authors wish to express their very sincere to reviewers for their valuable time spent reading this paper and for their valuable comment and suggestions. This work is supported by a fund from Universiti Teknologi MARA (Perak), Malaysia.

\section{References}

[1] Mat Yasin MH, Ishak A \& Pop I (2016), MHD heat and mass transfer flow over a permeable stretching/shrinking sheet with radiation effect. Journal of Magnetism and Magnetic Materials 407, 235-240.

[2] Azeman SNA \& Ishak A (2012), Heat transfer in thermal boundary layer flow over a stretching plate with radiation effect. Proceedings of the IEEE Business, Engineering and Industrial Applications Colloquium, pp 420-424

[3] Merkin JH \& Pop I (2011), The forced convection flow of a uniform stream over a flat surface with a convective surface boundary condition. Commun. Nonlinear Sci. Numerical Simul 16, 3602-3609.

[4] Shateyi S \& Mabood F (2015), MHD mixed convection slip flow near a stagnation-point on a nonlinearly vertical stretching sheet in the presence of viscous dissipation. Thermal Science 21(6B), 2731-2745.

[5] Merkin JH (1994), Natural - convection boundary - layer flow on a vertical surface with Newtonian heating. International Journal of Heat and Fluid Flow 15(5), 392-398.

[6] Lin C, Kao MJ, Tzeng GW, Wong WY, Yang J, Raikar RV \& Liu P LF (2015), Study on flow fields of boundary-layer separation and hydraulic jump during rundown motion of shoaling solitary wave. Journal of Earthquake and Tsunami 9(5), 1-33.

[7] Makinde OD \& Olanrewaju PO (2010), Buoyancy effects on the thermal boundary layer over a vertical flat plate with a convective surface boundary conditions. ASME Fluid Eng 132, 1-4.

[8] Merkin JH \& Mahmood T (1989), Mixed convection boundary layer similarity solutions: Prescribed wall heat flux. Zeitschrift Für Angewandte Mathematik Und Physik ZAMP 40(1), 51-68.

[9] Ishak A, Nazar R, \& Pop I (2008), Dual solutions in mixed convection flow near stagnation point on a vertical surface in a porous medium. International Journal of Heat and Mass Transfer 51(5-6), 1150-1155.

[10] Sharma R, Ishak A \& Pop I (2017), Dual solution of Unsteady separated stagnation-point flow in a nanofluid with suction: A finite element analysis. Indian Journal of Pure and Applied Physics 55(4), 275-283.

[11] Jusoh R, Nazar R \& Pop I (2017), Dual solutions of MHD threedimensional flow over a permeable stretching/shrinking surface with velocity slip and thermal radiation in a nanofluid. Journal of Computational and Theoretical Nanoscience 14(3), 1644-1652.

[12] Rossca AV, Roșca NC \& Pop I (2014), Note on dual solutions for the mixed convection boundary layer flow close to the lower stagnation point of a horizontal circular cylinder: Case of constant surface heat flux Sains Malaysiana 43(8), 1239-1247.

[13] Kimura S \& Bejan A (1983), The heatline visualization of convective heat transfer. Journal of Heat Transfer 105, 916-919.

[14] Lioua KHF, Oztop HF, Borjini MN \& Al-Salem K (2011), Second law analysis in a three dimensional lid-driven cavity. Int. Commun. in Heat and Mass Transfer 38, 1376-1383.

[15] Basak T, Pradeep TPV \& Roy S (2011), A complete heatline analysis on visualization of heat flow and thermal mixing during mixed convection in a square cavity with various wall heating. Industrial and Engineering Chemical Research 50, 7608-7630.

[16] Ishak A (2014), Dual solutions in mixed convection boundary layer flow: A stability analysis. International Journal of Mathematical, Computational, Physical and Quantum Engineering 8(9), 1131-1134.

[17] Cao L, Si X, Zhen L \& Pang H (2015), Lie group analysis for MHD effects on the convectively heated stretching porous surface with the heat source/sink. Boundary Value Problems 2015(1), 1-18.

[18] Chaudhary MA, Merkin JH (1993), The effect of blowing and suction on free convection boundary layers on vertical surfaces with prescribed heat flux. J. Engng. Math 27, 265-292.

[19] Ishak A (2014), Dual solutions in mixed convection boundary layer flow: A stability analysis. International Journal of Mathematical, Computational, Physical and Quantum Engineering 8(9), 1131-1134. 